\title{
The estimate and scaling of mass and energy fluxes from three different ecosystems in the Trentino Alto Adige/South Tyrol (Italy) region: Preliminary results from the Wheat Project.
}

\author{
Marco Falocchi ${ }^{1,2}$, Lorenzo Giovannini ${ }^{1}$, Luca Belelli Marchesini ${ }^{3}$, Damiano Gianelle ${ }^{3}$, Leonardo \\ Montagnani ${ }^{4}$, and Dino Zardi ${ }^{1,2}$ \\ ${ }^{1}$ University of Trento, Atmospheric Physics Group, Department of Civil Environmental and Mechanical Engineering, Italy \\ (marco.falocchi@unitn.it) \\ ${ }^{2}$ University of Trento, C3A - Center Agriculture Food Environment, Italy \\ ${ }^{3}$ Fondazione Edmund Mach, Department of Sustainable Agro-Ecosystems and Bioresources, Research and Innovation \\ Centre, Italy \\ ${ }^{4}$ Free University of Bolzano-Bozen, Faculty of Science and Technology, Italy
}

The estimate and parameterization of mass and energy fluxes exchanged in the atmospheric surface-layer, between the biosphere and the atmosphere, plays a key role in many disciplines, e.g. meteorology and atmospheric sciences, ecology and precision agriculture.

In mountain environments, crests lines tend to decouple the atmospheric processes close to the ground from those in the upper layers and deeply affect the penetration of solar radiation on the floors and the sidewalls of valleys. The consequent differential heating of the surface allows the onset of many local phenomena, such as thermally-driven flows and temperature inversions, with impacts on the regime of the exchanges. Indeed, the low-wind conditions, the wind interaction with landforms and the atmospheric stability control the turbulence and the development of submeso motions, i.e. those phenomena responsible for the diffusion and transport of substances, respectively.

The Wheat Project is a 2-year-long project, started on November 2018 and funded by the CARITRO Foundation ("Cassa di Risparmio di Trento e Rovereto", Italy), which aims at investigating the basic mechanisms responsible for biosphere-atmosphere exchanges in mountain areas, in order to improve their estimate and scaling.

Three datasets collected by long-term research infrastructures and composed of both biochemical and atmospheric quantities measured over different ecosystems were selected in the Trentino Alto Adige/South Tirol region (Italy). Data were measured at the research facility managed by the Free University of Bolzano/Bozen at Caldaro, over an apple orchard (220 m ASL, available period 2010-2018), and at the research facilities managed by the Fondazione Edmund Mach at Monte Lavarone, over a forest (1349 m ASL, available period 2000-2018) and at Viote del Monte Bondone, over an Alpine grassland (1553 m ASL, available period 2002-2018).

This contribution focuses on the pre-processing procedure adopted to identify representative 
periods for the analyses and on the methods implemented to retrieve turbulence parameters. In particular, the identification of the characteristic time-scales of small-scale turbulence is carried out through an application of the anisotropic analysis of turbulence, whereas the separation of the turbulence signal from low-frequency fluctuations is performed by implementing a recursive digital filter. Finally, some preliminary results regarding the estimate and the scaling of the turbulent fluxes of momentum, sensible heat, moisture and carbon dioxide are presented. 